\title{
Negative prompts aimed at maintaining eating independence
}

\author{
Alvisa Palese \\ University of Udine, Italy \\ Silvia Gonella \\ University of Torino, Italy \\ Tea Kasa and Davide Caruzzo \\ University of Udine, Italy
}

Mark Hayter and Roger Watson

University of Hull, UK

@SAGE

\begin{abstract}
Background: Psychological abuse of older people is difficult to recognise; specifically, nursing home residents have been documented to be at higher risk of psychological abuse during daily care, such as during feeding. Healthcare professionals adopt positive and negative verbal prompts to maintain residents' eating independence; however, negative prompts' purposes and implications have never been discussed to date.

Research aims: To critically analyse negative verbal prompts given during mealtimes as forms of abuse of older individuals and violation of ethical principles.

Research design: This is a secondary analysis of three cases of negative prompts that emerged in a large descriptive study based upon focus group methodology and involving 13 nursing homes and 54 healthcare professionals.

Participants and research context: This study included 3 out of $I 3$ nursing homes caring for residents with moderate/severe functional dependence in self-feeding mainly due to dementia; in these nursing homes, we conducted three focus groups and 13 healthcare professionals participated.

Ethical considerations: This study was conducted in accordance with the Human Subject Research Ethics Committee guidelines after being approved by the Review Board of the Trust.

Findings: With the intent of maintaining self-feeding independence, negative verbal prompts have been reported as being used by nursing home teams. By critically analysing these negative prompts, it turned out they could trigger intimidation, depression and anxiety and thus could be considered as forms of abuse; moreover, negative prompts can threaten the ethical principles of (I) autonomy using a paternalist approach, (2) beneficence and non-maleficence as with the intent to act in the best interests of residents (to maintain self-feeding independence) they are harmed in their dignity and (3) justice, given that residents who received negative prompts are treated differently from those who received positive prompts.
\end{abstract}

Corresponding author: Alvisa Palese, Department of Medical Sciences, University of Udine, Viale Ungheria 20, 330 I0 Udine, Italy. Email: alvisa.palese@uniud.it 
Discussion: Eating should be a pleasant experience with a positive impact on physiological, psychological and social well-being. However, negative prompting can lead to abuse and violation of basic ethical principles, destroying the healthcare professional resident and relative relationships strongly dependent on trust.

Conclusion: Negative verbal prompting must be avoided.

\section{Keywords}

Eating independence, elder abuse, ethical principles, feeding difficulties, mealtime assistance, nursing home, qualitative study, verbal prompting

\section{Introduction}

Over the past decade, elder abuse has been defined as the mistreatment of older people, which has been recently identified in five types, for example, physical, psychological, sexual, financial and neglect. ${ }^{1}$ Recognising elder abuse may be challenging and psychological abuse is among the subtlest forms to identify: intimidation, humiliation, disregard or causing fear are only some types of psychological abuse. ${ }^{2}$

The overall prevalence of elder abuse has been reported at approximately $10 \%,{ }^{1}$ although it is likely to be underestimated since cognitively impaired individuals can have difficulties in reporting abuse or may not be believed when they report it. For every recognised case, five or more have been estimated as being unreported. ${ }^{3}$ Specifically, given that most nursing home (NH) residents are admitted due to the consequences of cognitive impairments, in this setting, an increased risk of abuse has been reported also during daily basic care, such as support while eating. ${ }^{4}$

The prevalence of eating difficulties has been documented as high, involving $>85 \%$ of residents with advanced dementia. ${ }^{5,6}$ When residents' eating performance is initially impaired, healthcare professionals (HCPs) offer mealtime assistance in the form of verbal prompts and cues, positive reinforcements and appropriate encouragement. These have all been documented as interventions to maintain independence and the residents' active participation at mealtime rather than being a passive recipient of food. ${ }^{5}$ In advanced stages, when self-feeding worsens, partial (e.g. assisting the resident in holding the cup or tools) and total assistance (e.g. feeding the resident) are required. ${ }^{7,8}$

According to the evidence available, prompts delivered by HCPs aimed at maintaining or increasing eating performance have been categorised as follows: (1) tactile, for example, offering tactile prompts followed by immediately offering food; (2) motivational, for example, offering positive reinforcements when the resident completes the eating task, by saying 'That's right, good'; (3) role modelling, for example, positioning the resident with compromised eating abilities between residents who are able to feed themselves to provide examples as role models; (4) verbal, for example, instructing them to eat/drink or inviting questions as 'Why don't you try a bit of your soup?'; and (5) non-verbal, for example, opening containers, cutting meat. ${ }^{7-10}$

However, these prompts can assume the features of psychological abuse when they are delivered in a negative manner rather than providing positive reinforcements, for example, making the patient feel guilty for not being able to eat alone or threatening to withhold the reward at the end of the meal, as coffee or sweets, if all the food has not been eaten. ${ }^{2}$ Humiliation by infantilisation and threatening are examples of psychological abuses violating a resident's dignity and ethical principles. ${ }^{11}$ Their protection in NH settings relies to an extended degree on the behaviour, compassion and sensitivity of HCPs; therefore, they can be threatened when HCPs' authority prevails. ${ }^{11}$

To our best knowledge, an in-depth analysis of negative prompts offered during mealtimes aimed at stimulating eating independence has never been conducted with regard to their contribution to elder abuse 
and violation of ethical principles. With this intent, the principal purpose of this study was to contribute to the progression of the debate in this field by critically analysing the use of negative prompts as a form of psychological abuse to maintain self-feeding performance among NH residents with moderately severe to severe cognitive impairment and moderate to high dependence in eating.

\section{Background}

The concept of 'prompt' refers, according to its etymology, to 'incite' to do something. ${ }^{12}$ Prompts are offered before the action. Moreover, all interventions aimed at driving the action by anticipating what can happen if the action is appropriately performed (e.g. receiving a reward) can be considered a reinforcement. Prompts and reinforcements have been considered relevant among learning theories, mainly in the field of behaviourism. ${ }^{13,14}$

Negative reinforcements have been documented as aimed at removing, reducing, postponing or preventing certain stimuli. ${ }^{15}$ Negative reinforcers can generate two consequences among the residents, 'escaping' and 'avoiding': while the former refers to the attempt to remove or reduce a stimulus (e.g. when the resident starts to refuse to eat in the dining room during the meal due to his or her attempt to prevent negative prompts), the latter is aimed at preventing the occurrence of the stimulus (e.g. asking to eat in the bedroom and not in the dining room in order to avoid humiliations during meals). Moreover, while negative reinforcements consist in the attempt to remove something that can be unpleasant to a person, punishment is a process by which a consequence immediately follows a behaviour with the intent to decrease its future occurrence. ${ }^{16}$

Negative reinforcement has been considered intrusive as a punishment given that the presentation of the aversive stimulus is contingent on the absence, rather than the occurrence, of behaviour. ${ }^{16}$ Both concepts are widely used and debated in primary education, and according to Iwata, ${ }^{16}$ there is a need to conduct research with great care and under the appropriate conditions to determine how negative reinforcements might be used effectively and humanely, their limitations and their proper role within the larger realm of currently available educational strategies used to enhance learning.

Negative prompts may appear as psychological abuse when promoting residents' tendency to isolation during social activities as eating (e.g. residents prefer to eat in their room rather than with the others) to avoid punishment; moreover, they may also appear to threaten ethical principles that should guide HCP practice. Specifically, HCPs are required to assess the wishes and desires of each individual aiming at protecting his or her autonomy by avoiding 'paternalistic' patterns (respect for autonomy). Moreover, HCPs are required to act in the best interests of patients and their families'/carers' views and to not harm them (non-maleficence). Furthermore, HCPs are required to undertake decisions by ensuring fair distribution of scarce resources (distributive justice), by respecting individual rights (rights-based justice) and by respecting morally accepted laws (legal justice). ${ }^{17}$

\section{Methods}

\section{Study design}

This was a secondary analysis of negative cases ${ }^{18}$ that emerged in a large descriptive qualitative study ${ }^{19}$ based on focus group methodology ${ }^{20}$ performed in 2017. The COnsolidated criteria for REporting Qualitative research (COREQ) ${ }^{21}$ were considered here to report those methodological aspects related to the study design as summarised in Table 1. 
Table I. Analysis of the study conduction according to the COnsolidated criteria for REporting Qualitative research. ${ }^{2 I}$

No. of item Guide questions/description Strategies

Domain I: Research team and reflexivity

Personal characteristics

I. Interviewer/facilitator Which author(s) conducted the interview or focus group?

2. Credentials

What were the researcher's credentials? For example, PhD, MD

3. Occupation

4. Gender

5. Experience and training
What was their occupation at the time of the study?

Was the researcher male or female?

What experience or training did the researcher have?
A.P. conducted the interviews; T.K. participated by taking notes during the focus group conduction

A.P. was a PhD student and experienced in conducting qualitative studies T.K. was a clinical nurse, educated at the university level

A.P. was Associate Professor in Nursing Science, T.K. was a student

They were both female

They were trained in conducting interviews; specifically, A.P. was also supervised in interview conduction in previous research projects

Relationship with participants

6. Relationship established the interviewer

8. Interviewer characteristics
Was a relationship established prior to study commencement?

What did the participants know about the researcher?

What characteristics were reported about the interviewer or facilitator?
They met each other the first time in the day of the focus groups

They were informed on the study aims and on the role of the interviewer; no other data were shared

There were reported only their work position and their research lines No other data were shared

Domain 2: Study design

Theoretical framework

9. Methodological orientation and theory

Participant selection

10. Sampling

I I. Method of approach

12. Sample size

13. Non-participation
What methodological orientation was stated to underpin the study?

How were participants selected? How were participants approached?

How many participants were in the study?

How many people refused to participate None

and why?

\section{None}

There were undertaken a case study of negative cases according to Creswell $^{18}$

A purposeful sample was used

Participants were approached face-to-face by their $\mathrm{NH}$ nurse manager and invited to participate in the focus group

In the primary study, there were 54 participants; in the focus groups where the negative cases emerged, I 3 participants were involved 
Table I. (continued)

$\begin{array}{lll}\text { No. of item } \quad \text { Guide questions/description } & \text { Strategies }\end{array}$

Setting

14. Setting of data collection Where was the data collected? For example, home, clinic, workplace

15. Presence of nonparticipants

16. Description of sample

Data collection

17. Interview guide

18. Repeat interviews

19. Audio/visual recording

20. Field notes

21. Duration

22. Data saturation

23. Transcripts returned
Was anyone else present besides the participants and researchers?

What are the important characteristics of the sample?

Were questions, prompts, guides provided by the authors? Was it pilot tested?

Were repeat interviews carried out? If yes, how many?

Did researchers use audio/visual recording to collect the data?

Were field notes made during and/or after the focus group?

What was the duration of the interviews or focus group?

Was data saturation discussed?

Were transcripts returned to participants for comment and/or correction?
The data were collected in the $\mathrm{NHs}$, in appropriate environment protected by interruptions and/or sources of disturbance

None

Characteristic of the participants has been collected and is reported in Table 2

The questions were defined by authors and pilot tested in an $\mathrm{NH}$ not involved in the final study

No repeated interviews were performed

The focus groups were all audio-recorded

There were collected in-the-field notes

In those focus groups were the negative cases NA emerged, the duration was from 55 to $65 \mathrm{~min}$

Sessions of member checking ${ }^{22}$ of data extracted have been performed by returning to two out of three $\mathrm{NH}$ focus groups

Domain 3: Analysis and findings

Data analysis

24. Number of data coders

25. Coding tree description

26. Derivation of themes

27. Software

28. Participant checking
How many data coders coded the data? NA

Did authors provide a description of the NA coding tree?

Were themes identified in advance or NA derived from the data?

What software, if applicable, was used NA to manage the data?

Did participants provide feedback on the findings?
Sessions of member checking ${ }^{22}$ of data extracted have been performed by returning to two out of three $\mathrm{NH}$ focus groups

\section{Reporting}

29. Quotations presented

30. Data and findings consistent
Were participant quotations presented to illustrate the themes/findings?

Was there consistency between the data presented and the findings?
NA

\author{
(e.g. $\mathrm{NH} \mathrm{I})$
}

(continued) 
Table I. (continued)

\begin{tabular}{lll}
\hline No. of item & Guide questions/description & Strategies \\
\hline 31. Clarity of major themes & $\begin{array}{c}\text { Were major themes clearly presented } \\
\text { in the findings? }\end{array}$ & NA \\
32. Clarity of minor themes & $\begin{array}{c}\text { Is there a description of diverse cases or } \\
\text { discussion of minor themes? }\end{array}$ & NA \\
\hline
\end{tabular}

$\mathrm{NH}$ : nursing home; HCP: healthcare professional; NA: not applicable for the purpose of this case study.

\section{Setting and participants}

The primary study involved 13 NHs that were regulated by the Regional Health Services and located in a rural area of the north-east of Italy. ${ }^{19}$ Residents were admitted to these NHs with moderate/severe functional dependence in need of feeding care due to different health conditions, mainly dementia. ${ }^{23}$

Specifically, a purposeful sample ${ }^{24}$ of HCPs in each NH (1) involved on a daily basis in assisting residents at mealtimes, (2) working in the NH for the past 6 months and (3) willing to participate, were invited to be part of the focus group formed in each NH. HCPs $(n=54)$ were involved in the primary study $;^{19}$ in this secondary analysis, findings from 13 HCPs working in three NHs were analysed.

\section{Data collection process}

After having observed (A.P., T.K.) the HCPs during mealtime assistance in the dining room of each involved $\mathrm{NH}$ and collecting in-the-field notes regarding behaviour and verbal or non-verbal interventions adopted to maintain eating independence, a purposeful sample of HCPs was selected by the NH nurse manager. A focus group methodology ${ }^{25}$ was used to collect data; thus, 13 focus group interviews were conducted by following a specific guide (available from authors) developed by researchers: after collecting some demographic and professional data, a set of open-ended questions were asked aimed at stimulating the detection of interventions performed on a daily basis, aimed at maintaining residents' eating performance. Thus, by reporting some interventions witnessed by researchers during their lunchtime observation, the discussion was further continued with the purpose of collecting all interventions delivered. Furthermore, the interventions that emerged in the previous focus groups were reported with the intent to introduce new insights and data into those already collected. ${ }^{26}$

A senior researcher (A.P.) conducted the data collection process with the support of a junior researcher (T.K.) who collected notes during the interview; the focus groups were all conducted in appropriate $\mathrm{NH}$ environments facilitating interactions among participants. The focus groups were on average $1 \mathrm{~h}$ in length (from 45 to $130 \mathrm{~min}$ ); they were audio-recorded and then verbatim-transcribed. Aiming at ensuring data dependability, ${ }^{22}$ the focus groups were numbered (e.g. focus group Nursing Home 5, NH 5).

\section{Negative case identification}

From the verbatim transcriptions, there were three negative cases identified in three different NHs that did not support or contradict the patterns of positive prompts with regard to self-feeding that emerged from the data analysis. ${ }^{18}$ These cases were preliminarily categorised as 'verbal prompts' but given (1) their negative intention, thus not intended to reward or support the resident; ${ }^{7-10}$ and (2) their potential negative impact on residents (e.g. fear), ${ }^{2}$ they were selected and extracted.

Moreover, in-the-field notes collected during the mealtime observation phase were all also scrutinised by two researchers (A.P., S.G.) to check whether the cases emerged in the focus groups were also witnessed as 
actual interventions; in the field notes, no data were reported. Furthermore, aiming at ensuring data consistency, sessions of focus group member checking ${ }^{22}$ of extracted data were performed by returning to two NH focus groups, selected casually, out of three. These agreed with the data extracted, which was then considered valid. Thus, these negative cases were contextualised by describing their profile at the following levels:

a. NHs levels - as the number of beds and the number of residents living in each;

b. Resident levels - by reporting age, gender, the degree of functional dependence as measured by the Barthel Index score (from $0=$ total dependence to $100=$ total independence), ${ }^{27}$ the degree of the cognitive impairment as measured by the Cognitive Performance Scale (CPS, from $0=$ intact to $6=$ severe impairment; scores $\geq 4$ indicate moderate/severe cognitive impairment) ${ }^{28}$ and the selffeeding impairments as measured by the Edinburgh Feeding Evaluation in Dementia scale (EdFED), ${ }^{29,30}$ ranging from 0 (any difficulties) to 20 (complete dependence in self-feeding);

c. Focus group levels - by reporting participants' ages, gender, professional profiles and years of clinical experience in the $\mathrm{NH}$.

While at the NHs and at the focus group levels, data were collected by the researchers involved, data at the residents' level were collected through observation, interview or clinical record access, by trained Registered Nurses (RNs) responsible for the resident's care in the facilities, using the abovementioned tools.

\section{Negative case critical analysis}

In the preliminary phase, the Lachs and Pillemer's1 framework was adopted to identify and discuss elder abuse. According to this framework, elder abuse can be physical, psychological or verbal, sexual, financial and neglect. Manifestations of abuse change according to the type of abuse: (1) physical abuse should be suspected with abrasions, lacerations, bruises, fractures, burns, pain, depression or delirium; (2) psychological abuse is suspected when verbal abuse is directly observed or with subtle signs of intimidation, such as deferring questions to some caregivers, producing isolation of the patient from both previously trusted family and/or friends, depression and/or anxiety; (3) sexual abuse is hypothesised in the presence of lacerations in the anogenital area or abdomen, newly acquired sexually transmitted disease or urinary tract infection; (4) nonadherence to medication regimen, malnutrition, weight loss or inability to pay for medicine may be manifestations of financial abuse; while (5) decubitus ulcers, poor hygiene and dehydration may indicate neglect. Thus, according specifically with the manifestations reported in point 2 , the negative verbal prompts were considered as a form of psychological abuse.

Then, the three cases identified were analysed in depth according to the ethical principles of autonomy, beneficence/non-maleficence and justice. ${ }^{31}$ Furthermore, the feminist ethic of care model based upon relationships, responsibility, trust, loyalty and sensitivity was also used to analyse the cases. ${ }^{32}$ According to this framework, HCPs should demonstrate: (1) moral attention, paying attention to the complexity of the situation and get involved; (2) sympathetic understanding; (3) relationship awareness; and (4) harmony, by ensuring balance in the relationship. ${ }^{32}$

Negative cases were analysed individually and then collectively by all authors according to the abovementioned frameworks.

\section{Ethical considerations}

The Review Board of the reference Health Care Trust of the NHs approached approved the study protocol. Participants were informed on the study purpose and they were free to participate and to withdraw from the 
Table 2. Nursing homes, residents and focus group participants' profiles.

\begin{tabular}{|c|c|c|c|}
\hline $\mathrm{NH}$ & $\mathrm{NH}$. I & $\mathrm{NH}$ n. 5 & NH n. 7 \\
\hline \multicolumn{4}{|l|}{ NH profile } \\
\hline Bedsides, $\mathrm{n}$ & 49 & 102 & 87 \\
\hline $\begin{array}{l}\text { Residents living in the } \mathrm{NH} \text { at the } \\
\text { moment of the study, } \mathrm{n}\end{array}$ & 46 & 95 & 80 \\
\hline \multicolumn{4}{|l|}{ Resident profile } \\
\hline Age, in years (SD) & $88.6(8.5)$ & $81.3(11.8)$ & $86.0(8.8)$ \\
\hline F/M & $36 / 10$ & $70 / 2 I^{\mathrm{a}}$ & $57 / 23$ \\
\hline Barthel Index $(0-100)(95 \% \mathrm{Cl})^{\mathrm{b}}$ & $22.86(|4.35-3| .37)$ & $27.06(20.54-33.58)$ & $37.96(29.67-46.25)$ \\
\hline CPS (0-6) $(95 \%$ Cl) & $3.02(2.38-3.67)$ & $3.13(2.7 \mid-3.54)$ & $2.59(2.09-3.09)$ \\
\hline CPS $>4^{\mathrm{c}}(\%)$ & $15(35.7)$ & $32(39.5)$ & $28(34.1)$ \\
\hline EdFED $(0-20)(95 \% \mathrm{Cl})^{\mathrm{d}}$ & 7.29 (4.70-9.87) & $1.95(1.38-2.52)$ & $1.99(|| 7-.2.8 I)$ \\
\hline \multicolumn{4}{|l|}{ Focus group participants' profile } \\
\hline Participants role, $\mathrm{n}$ & 2 NAs, I PHYS & 3 NAs, 2 RNs, I ED & 2 RNs, 2 NAs \\
\hline $\mathrm{F} / \mathrm{M}$ & $3 / 0$ & 6/I & $3 / 1$ \\
\hline Age, in years $(S D)$ & $39.3(10.0)$ & $50.1(5.2)$ & $37.0(16.7)$ \\
\hline Experience, in years (SD) & $10.0(8.0)$ & $22.8(9.1)$ & $10.3(3.4)$ \\
\hline
\end{tabular}

$\mathrm{Cl}$ : confidence interval; CPS: Cognitive Performance Scale; ED: professional educator; EdFED: Edinburgh Feeding Evaluation in Dementia scale; F: female; M: male; NH: nursing home; NA: nurse assistant; PHYS: physiotherapists; RN: Registered Nurse; SD: standard deviation.

${ }^{a}$ Four missing data.

${ }^{b}$ Lower scores indicate higher dependence in activity of daily living.

'Moderately severe to very severe cognitive impairment.

${ }^{\mathrm{d}}$ Higher scores indicate higher dependence in self-feeding.

study at any time. They were asked to sign the consent form before the start of study where they also agreed to be audio-recorded; during the data analysis, researchers anonymised the name of both participants and NHs.

\section{Findings}

\section{Negative prompts: cases emerged}

With the intent of maintaining or promoting eating independence, in three NHs, the staff used three forms of negative prompts reported as follows:

(1) Case 1: 'If the resident does not eat alone, we tell him or her that we will call his/her daughter'. (NH n. 7)

(2) Case 2: 'If the resident does not self-feed, we tell him or her that we will not give them sweets and/or coffee at the end of the lunch'. (NH n. 5)

(3) Case 3: 'Sometimes residents are stimulated by saying... It is really a shame to feed you given that you can still be independent...' (NH n. 1)

As reported in Table 2, these cases emerged in three NHs equipped with a range of beds, from 49 to 102 , and reporting bed occupancy rates $>90 \%$.

Residents living in these NHs were on average $>80$ years old and mainly female $(>60 \%)$. Moreover, in all NHs, the degree of dependence as measured with the Barthel Index was $<40$ out of 100 , thus suggesting a complete or partial functional dependence; with regard to self-feeding difficulties as measured with EdFED, 
on average, residents living in NH 1 had a high dependence for self-feeding, while those living in NHs 5 and 7 had a moderate dependence. Furthermore, three quarters of residents were affected by moderately severe to very severe cognitive impairment according to the CPS measures as reported in Table 2.

The focus groups in all NHs were multi-professional based, mainly composed by female nurse assistants and RNs with long experience (on average $>10$ years) in caring older people in NHs (Table 2).

\section{Negative prompts as a form of elder abuse}

According to the Lachs and Pillemer's ${ }^{1}$ framework, Case 1 (NH n. 7) expresses a sort of intimidation and can be considered a form of verbal/psychological abuse. Also, Cases 2 and 3 (NH n. 5 and NH n. 1), where depression and anxiety can be triggered by the negative prompts, can be classified as forms of verbal/ psychological elder abuse.

\section{Negative prompts as a form of violation of ethical principles}

According to Beauchamp and Childress ${ }^{31}$ different ethical principles are threatened in the cases selected. In all cases, a paternalistic approach seems to emerge, thus threatening the principle of autonomy; in the intent of serving the best interests of patients, which is substantially to maintain eating independence (= principle of beneficence), residents are harmed in their dignity by negative prompts. Furthermore, the principle of justice is also threatened given that (1) residents who received negative prompts are treated differently with respect to those who received positive prompts, (2) residents suffering from dementia have an increased risk of receiving negative prompts as compared to those cognitively unimpaired and (3) these negative prompts may have been given in order to implicitly apply pressure on the residents to reduce time spent in feeding care due to rationed nursing care, ${ }^{33}$ for example, residents receiving negative prompts can undertake avoidance behaviour, thus becoming more quick in eating.

\section{Discussion}

We have selected negative prompts cases used by the staff to enhance eating performance in 3 out of 13 NHs. The healthcare staff had been already documented to adopt a wide range of strategies to promote independence in eating, including prompting. ${ }^{5}$ Positive prompts have been associated with the retention of eating independence, ${ }^{7,9}$ fluid intake between meals, ${ }^{10}$ oral intake during meals ${ }^{8}$ and weight maintenance. ${ }^{9}$ However, to our best knowledge, negative prompts have never been discussed to date.

\section{Negative prompts as a form of abuse}

Resident's independence at mealtime has been reported as in indicator of quality of life ${ }^{34}$ with a recently renovated appeal to maintain residents' performance in eating as much as possible. ${ }^{5,35}$ International guidelines and position papers on nutrition in dementia have also recommended to include in the usual care all efforts to enhance oral feeding. ${ }^{36-38}$ Thus, HCPs are used to employing verbal prompts to stimulate the resident to recall movements and their necessary sequences to eat independently; moreover, HCPs are used to adopting motivational prompts congratulating residents when they complete the eating task. However, according to our findings, HCPs also adopt negative prompting. By intimidating and triggering distress, anxiety and depression,, 4 as single or repeated acts occurring within the caring relationship where there is an expectation of trust, ${ }^{39}$ negative prompting can be considered a form of elder abuse.

The mechanism involved in the three cases that emerged seems to be different: in Case 1 ('If the resident does not eat alone, we tell him/her that we will call his/her daughter'), there is subtle mistreatment in the form of intimidation; ${ }^{2}$ in Case 2 ('If the resident does not self-feed, we tell him/her that we will not give 
them sweets and/or coffee at the end of lunch'), NH residents are threatened by something pleasant that is not offered as a reward, but as a deprivation that can become a punishment; 4 while in Case 3 ('Sometimes residents are stimulated by saying... It is really a shame to feed you given that you can still be independent'), residents are made to feel guilty for not being able to eat alone, thus causing them anguish and distress. ${ }^{2}$

NHs have already been recognised as places at greater risk of abuse due to the increasing number of cognitively impaired older adults who spend parts of their remaining life there. ${ }^{40}$ As with other forms of abuse, negative prompts should be prevented and avoided; they can be difficult to discover because they occur inside of the dyad (resident and HCP) and should be taken into consideration in those studies aimed at measuring the occurrence of the phenomenon of verbal/psychological abuse as well as in educational strategies enacted to prevent all forms of abuse.

\section{Negative prompts as a form of violation of ethical principles}

Eating should be a pleasurable experience with a positive impact on physiological, psychological and social well-being. ${ }^{34}$ Therefore, establishing clear boundaries between positive (e.g. encouraging or rewarding) and negative prompting (e.g. punishing) is essential to identify the ethical nature of eating interventions beyond the beneficent scope of increasing residents' nutritional intake.

In the three cases emerged, HCPs were likely guided by the ethical principle of beneficence, thus believing to act in the resident's interest. ${ }^{31}$ Their scope of practice implies a set of tasks as ensuring nutrition; thus, they can have adopted negative prompts to fulfil their task obligations. However, the principle of beneficence means that unless there is a sufficient reason not to, the obligation is to guarantee interventions that are likely to provide more good than harm; ${ }^{41}$ differently, by adopting negative prompting, residents may feel humiliated, intimidated or guilty or experience forms of psychological distress such as fear, anxiety or depression. ${ }^{1}$ Thus, HCPs have several good reasons not to adopt negative prompts (e.g. avoiding resident's distress).

Caring with reference to the ethics of duty has to prevent all forms of compromise of a resident's psychological well-being according to the principle of non-maleficence defined as the obligation not to perform interventions that are likely to produce more harm than good. ${ }^{41}$ Therefore, the principle of non-maleficence suggests that negative prompts have to be avoided as they place residents at higher risk of distress.

Furthermore, according to the principle of justice, patients with the same needs should not be treated differently; ${ }^{31}$ thus, it should not be tolerable that some individuals (such as those with dementia) are exposed to positive and others to negative reinforcement(s) to reach the same goal, namely, maintaining independence in eating. Discovering the reasons of these different treatments is strongly recommended with the aim of preventing their occurrence, for example, increased paternalistic approaches with those who cannot be independent, such as older people with dementia, or accelerating mealtime processes due to implicit rationing of $\mathrm{NH}$ nursing care because of economical restraints.

It has been already documented that ensuring care to older adults affected by cognitive impairments by respecting all ethical principles can be challenging. ${ }^{42}$ As dementia progresses, the person provides minimal contributions; they become progressively incapable of making decisions concerning their care and health and to express their needs; ${ }^{43}$ symptoms such as resistance, restlessness and aggression have been documented as being common during nursing care, as eating assistance leaves HCPs in an ethically challenging situation between respecting resident's self-determination that leads to consequent malnutrition and forcing them to eat. ${ }^{44}$ However, the principle of autonomy translates into the principle of informed consent and to affirm autonomy treatment preferences needs to be discussed with residents and their relatives and documented in their records; 31 thus, HCPs assume a greater responsibility. HCPs may adopt different strategies 
to promote the patient's right to self-determination despite cognitive decline, for example, assessing the patient's wishes using close questions, involving relatives in the discussion making sure that their preferences were in accordance with the resident's preferences or by allowing relatives to remind them about past preferences. $^{45}$

Furthermore, according to the ethics of care, ${ }^{32}$ in the three cases that emerged, HCPs did not pay moral attention to the complexity of the situation and the adoption of negative prompts suggests that eating assistance was perceived as a routine task among others. Similarly, in accordance with previous studies, ${ }^{46}$ HCPs failed to recognise the importance of the psychosocial aspects of mealtimes and, given that all cases emerged from focus groups and not from singular interviews, factors at the NH levels could have played a strong influence in shaping mealtime interventions, for example, NH policies are often task-oriented aimed at guaranteeing adequate caloric intake, ${ }^{47}$ thus threatening other dimensions of mealtime.

Moreover, the three cases suggest that HCPs lacked sympathetic understanding since they did not reflect upon the unpleasant feelings that the negative prompting could induce, making the residents feel guilty for not being able to eat alone or fear their relatives' disappointed reactions when informed about their refusal of food. All interactions between human beings involve a basic trust; having trust means having power. ${ }^{48}$ In all cases, HCPs emerged as powerful over the residents, thus leading to disruption of the relationship. Simultaneously, HCPs misused their power over family caregivers by threatening to call the relative if the resident does not eat independently, an act that infantilises the person and interferes in the familiar relationship. Thus, the balance between the HCP and the care recipient and their relatives is disrupted with a loss of harmony.

\section{Study strengths and limitations}

We analysed three deviant cases that freely emerged during focus group sessions, during which there was the development of a great sense of trust between participants and researchers and the latter never expressed any judgements or personal opinions during data collection. However, according to the in-the-field notes collected in the first stage of the study, these cases were not witnessed in the real world during the lunchtime observation performed before the focus groups in each $\mathrm{NH}$. Therefore, their tonality and volume (e.g. shout) as well as their actual implementation have not been evaluated and scrutinised.

By conducting an interview via focus group, we have documented events through the knowledge of HCPs that drives them in daily practice; ${ }^{49}$ however, more studies aimed at detecting different forms of negative prompts as adopted at the bedside are required to understand in depth their implications both in elder abuse and in the violation of ethical principles.

Only three cases were analysed that emerged in 3 focus groups out of 13 conducted across different NHs. Therefore, the cases under study were limited and more studies are needed to discover the variability of negative prompts used in NHs as well as their frequency of use.

\section{Conclusion and implications}

Verbal reinforcements have been promoted for their positive effects in maintaining eating independence; however, some prompts that are negatively directed (e.g. punishing or increasing stress and anxiety) instead of positively directed (based upon rewards) are used during mealtimes. According to their manifestation, negative prompts can be considered a verbal/psychological abuse that must be prevented and avoided; moreover, negative prompts can violate ethical principles of care. It is undeniable that pursuing resident's self-feeding independence has a beneficence scope; however, threatening the trust of residents and their relatives, for example, by adopting negative prompts, can disrupt the relationship with both of them, thus violating the respect of ethical responsibilities towards older people who are in need. Therefore, verbal 
prompting should always be positively directed and negative prompts avoided to prevent abuse and violation of ethical principles.

Since the negative prompts emerged only within focus groups, further investigation of the role of the $\mathrm{NH}$ environment (i.e. culture) in shaping mealtime interventions is suggested, with a greater attention for reaching adequate nutritional intake rather than ensuring that the pleasure for a social activity as eating is maintained. Therefore, this analysis provides a basis for discussions among HCPs about aspects of their work of which they might not have previously been aware. In turn, such discussions may result in a change in the attitudes and behaviours of HCPs in promoting independence in eating among NH residents and the adoption of other strategies in place of negative prompts to overcome self-feeding difficulties, particularly in those residents with cognitive impairment. Improvements in awareness, collaboration, and open discussion of concerns, difficulties, and values, beliefs are necessary for improvement.

However, an in-depth understanding of why negative prompts are offered, as well as when and under what mechanisms they are originated and included as a part of the usual care is strongly recommended. Furthermore, negative prompts should be taken into consideration in studies aimed at measuring the occurrence of verbal/psychological abuse.

\section{Conflict of interest}

The author(s) declared no potential conflicts of interest with respect to the research, authorship and/or publication of this article.

\section{Funding}

The author(s) received no financial support for the research, authorship and/or publication of this article.

\section{ORCID iD}

Alvisa Palese (1) https://orcid.org/0000-0002-3508-844X

\section{References}

1. Lachs MS and Pillemer KA. Elder abuse. N Engl J Med 2015; 373(20): 1947-1956.

2. Marshall KA and Hale D. Elder abuse. Home Healthc Now 2018; 36(1): 51-52.

3. Bonnie R and Wallace R. Elder mistreatment: abuse, neglect and exploitation in an aging America. Washington, DC: National Academies Press, 2003.

4. Dong X, Chen R and Simon MA. Elder abuse and dementia: a review of the research and health policy. Health Aff 2014; 33(4): 642-649.

5. Liu W, Galik E, Boltz M, et al. Optimizing eating performance for older adults with dementia living in long-term care: a systematic review. Worldviews Evid Based Nurs 2015; 12(4): 228-335.

6. Mitchell SL, Teno JM, Kiely DK, et al. The clinical course of advanced dementia. N Engl J Med 2009; 361(16): $1529-1538$.

7. Coyne ML and Hoskins L. Improving eating behaviors in dementia using behavioral strategies. Clin Nurs Res 1997; 6(3): 275-290.

8. Simmons SF and Schnelle JF. Individualized feeding assistance care for nursing home residents: staffing requirements to implement two interventions. J Gerontol A Biol Sci Med Sci 2004; 59(9): M966-M973.

9. Van Ort S and Phillips LR. Nursing intervention to promote functional feeding. J Gerontol Nurs 1995; 21(10): 6-14.

10. Simmons SF, Alessi $\mathrm{C}$ and Schnelle JF. An intervention to increase fluid intake in nursing home residents: prompting and preference compliance. J Am Geriatr Soc 2001; 49(7): 926-933.

11. Rehnsfeldt A, Lindwall L, Lohne V, et al. The meaning of dignity in nursing home care as seen by relatives. Nurs Ethics 2014; 21(5): 507-517. 
12. Alberto AA and Troutman AC. Applied behaviour analysis for teacher. 6th ed. Englewood Cliffs, NJ: Prentice Hall, 2003.

13. Hilgard ER and Bower GH. Theories of learning. Englewood Cliffs, NJ: Prentice Hall, 1975.

14. Skinner BF. Science and human behavior. New York: Free Press, 1953.

15. Hineline PN. Negative reinforcement and avoidance. In: Honig WK and Staddon ER (eds) Handbook of operant behavior. Englewood Cliffs, NJ: Prentice Hall, 1977, pp. 364-414.

16. Iwata BA. Negative reinforcement in applied behavior analysis: an emerging technology. J Appl Behav Anal 1987; 20(4): 361-378.

17. Gillon R. Medical ethics: four principles plus attention to scope. Br Med J 1994; 309(6948): 184-188.

18. Creswell JW. Qualitative inquiry and research design choosing among five traditions. Thousand Oaks, CA: Sage, 1998.

19. Palese A, Bressan V, Kasa T, et al. Interventions maintaining eating independence in nursing home residents: a multicentre qualitative study. BMC Geriatrics 2018; 18:292.

20. Vaismoradi M, Jordan S, Turunen $\mathrm{H}$, et al. Nursing students' perspectives of the cause of medical errors. Nurse Educ Today 2014; 34(3): 434-440.

21. Tong A, Sainsbury P and Craig J. Consolidated criteria for reporting qualitative research (COREQ): a 32-item checklist for interviews and focus groups. Int J Qual Health Care 2007; 19(6): 349-357.

22. Morse JM. Critical Analysis of strategies for determining rigor in qualitative inquiry. Qual Health Res 2015; 25(9): 1212-1222.

23. Palese A, Grassetti L, Bandera D, et al. High feeding dependence prevalence in residents living in Italian nursing homes requires new policies: findings from a regionally based cross-sectional study. Health Policy 2018; 122(3): 301-308.

24. Patton MQ. Qualitative research \& evaluation methods: integrating theory and practice. 4th ed. Thousand Oaks, CA: Sage, 2015.

25. Krueger RA and Casey MA. Focus groups: a practical guide for applied research. 5th ed. Thousand Oaks, CA: Sage, 2014.

26. Sandelowski M. Whatever happened to qualitative description? Res Nurs Health 2000; 23(4): 334-340.

27. Mahoney FI and Barthel DW. Functional evaluation: the Barthel Index. Md State Med J 1965; 14: 56-61.

28. Morris JN, Fries BE, Mehr DR, et al. MDS cognitive performance scale. J Gerontol 1994; 49(4): 174-182.

29. Watson R, Bagnasco A, Catania G, et al. The Edinburgh Feeding Evaluation in Dementia Scale: a longitudinal study in nursing home residents. Dement Geriatr Cogn Disord 2017; 44(3-4): 196-202.

30. Bagnasco A, Watson R, Zanini M, et al. Preliminary testing using Mokken scaling of an Italian translation of the Edinburgh Feeding Evaluation in Dementia (EdFED-I) scale. Appl Nurs Res 2015; 28(4): 391-396.

31. Beauchamp T and Childress J. Principles of biomedical ethics. 4th ed. New York: Oxford University Press, 2001.

32. Gilligan C. In a different voice: psychological theory and women's development. Cambridge, MA: Harvard University Press, 1982.

33. Basso I, Bonaudo M, Dimonte V, et al. Le Missed Care negli ospiti delle residenze sanitarie per anziani: risultati di uno studio pilota [The missed care in Nursing Homes: a pilot study]. Assist Inferm Ric 2018; 37(3): 136-143.

34. Keller HH, Schindel Martin L, Dupuis S, et al. Mealtimes and being connected in the community-based dementia context. Dementia 2010; 9(2): 191-213.

35. Abdelhamid A, Bunn D, Copley M, et al. Effectiveness of interventions to directly support food and drink intake in people with dementia: systematic review and meta-analysis. BMC Geriatr 2016; 16: 26.

36. Volkert D, Chourdakis M, Faxen-Irving G, et al. ESPEN guidelines on nutrition in dementia. Clin Nutr 2015; 34(6): 1052-1073.

37. Barrocas AGeppert C, Durfee SM, et al. A.S.P.E.N. ethics position paper. Nutr Clin Pract 2010; 25(6): 672-679. 
38. American Geriatrics Society Ethics Committee and Clinical Practice and Models of Care Committee. American Geriatrics Society feeding tubes in advanced dementia position statement. J Am Geriatr Soc 2014; 62(8): $1590-1593$.

39. World Health Organization. Chapter 5: abuse of the elderly. In: Krug E, Dahllberg L and Mercy J, et al. (eds) World report on violence and health, http://www.who.int/violence_injury_prevention/violence/global_campaign/en/ chap5.pdf (2002, accessed 16 July 2018).

40. Bone AE, Gomes B, Etkind SN, et al. What is the impact of population ageing on the future provision of end-of-life care? Population-based projections of place of death. Palliat Med 2018; 32(2): 329-336.

41. Garrett TM, Baillie HW and Garrett RM. Health care ethics: principles and problems. Englewood Cliffs, NJ: Prentice Hall, 1993.

42. Schwiebert V, Myers J and Dice C. Ethical guidelines for counsellors working with older adults. J Couns Dev 2000; 78(2): 123-129.

43. Amella EJ, Grant AP and Mulloy C. Eating behavior in persons with moderate to late-stage dementia: assessment and interventions. J Am Psychiatr Nurses Assoc 2008; 13(6): 360-367.

44. Haibo X, Shifu X, Pin NT, et al. Prevalence and severity of behavioral and psychological symptoms of dementia (BPSD) in community dwelling Chinese: findings from the Shanghai three districts study. Aging Ment Health 2013; 17(6): 748-752.

45. Österholm JH, Taghizadeh Larsson A and Olaison A. Handling the dilemma of self-determination and dementia: a study of case managers' discursive strategies in assessment meetings. J Gerontol Soc Work 2015; 58(6): 613-636.

46. Dunn $\mathrm{H}$ and Moore T. 'You can't be forcing food down 'em': nursing home carers' perceptions of residents' dining needs. J Health Psychol 2016; 21(5): 619-627.

47. Gibbs-Ward AJKeller HH. Mealtimes as active processes in long-term care facilities. Can J Diet Pract Res 2005; 66(1): 5-11.

48. Løgstrup KE. The ethical demand. Paris: University of Notre Dame, 1997, 320 pp.

49. McAdam R, Mason B and McCrory J. Exploring the dichotomies within the tacit knowledge literature: towards a process of tacit knowing in organizations. J Knowledge Manag 2007; 11: 43-59. 